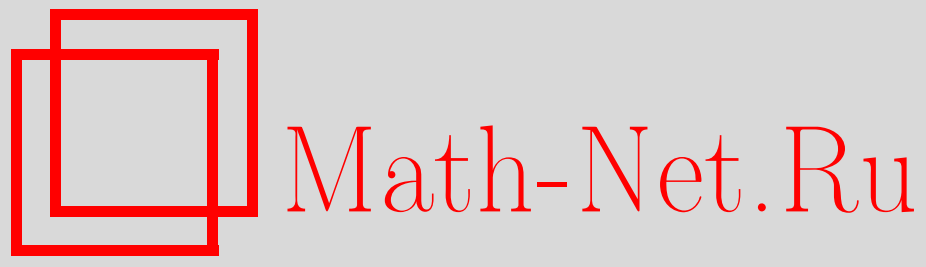

В. В. Рыжиков, Ж.-П. Тувено, Дизъюнктность, делимость и квазипростота сохраняющих меру действий, Функи. анализ и его прил., 2006, том 40, выпуск $3,85-89$

DOI: https://doi.org/10.4213/faa749

Использование Общероссийского математического портала Math-Net.Ru подразумевает, что вы прочитали и согласны с пользовательским соглашением

http://www. mathnet.ru/rus/agreement

Параметры загрузки:

IP: 54.80 .73 .141

26 апреля 2023 г., 16:05:33

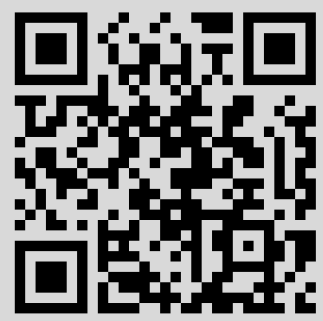


отображения, и, значит, оно является гомеоморфным вложением. Полупростота алгебры $\mathscr{A}$ следует из теоремы единственности преобразования Фурье в $L_{1}(G)$.

Если $G$ дискретна, то индикаторная функция нуля $\varepsilon$ содержится в $\mathscr{A}$, так как $\|\varepsilon\|_{p, w}=w_{0} \lambda(0)^{1 / p}<\infty ; \varepsilon$ является единицей относительно сверточного умножения. Если, обратно, в $\mathscr{A}$ есть единица $\varepsilon$, то ее преобразование Гельфанда $\hat{\varepsilon} \in C_{0}(\operatorname{Sp} \mathscr{A})$ равно всюду 1 ; соответственно $1=\left.\hat{\varepsilon}\right|_{\widehat{G}} \in C_{0}(\widehat{G})$. Но это возможно только тогда, когда группа $\widehat{G}$ компактна, т. е. $G$ дискретна.

\title{
ЛитературА
}

[1] S. Saeki, Illinois J. Math., 34:3 (1990), 614-627. [2] Н. К. Никольский, Трудъь МИАН, 120 (1974). [3] S. Grabiner, Amer. J. Math., 97:1 (1975), 16-42. [4] R. Kerman, E. Sawyer, Stud. Math., 108:2 (1994), 103-126. [5] A. Benazzouz, A. El Kinani, Bull. Belg. Math. Soc. Simon Stevin, 10:1 (2003), 49-57. [6] S. A. Shkarin, Yu. N. Kuznetsova, J. Math. Sci. (New York), 131:6 (2005), 6112-6119. [7] H. G. Dales, in: Radical Banach algebras and automatic continuity (Proc. Conf., Long Beach 1981), Lecture Notes in Math., vol. 975, 1983. [8] Э. Хьюитт, К. Росс, Абстрактный гармонический анализ, т. I, II, Наука, М., 1975.

Московский институт электроники и математики e-mail: jkuzn@mccme.ru

\section{Дизъюнктность, делимость и квазипростота сохраняющих меру действий*}

\author{
(c) 2006. В. В. Рыжиков, ЖК.-П. Тувено
}

Знание структуры самоприсоединений динамической системы с инвариантной мерой дает информацию о факторах и централизаторе тензорных произведений системы, оказывается полезным в построении контрпримеров в эргодической теории и в исследовании проблемы В. А. Рохлина о кратном перемешивании. Из отсутствия у перемешивающей системы нетривиальных самоприсоединений, которые обладают свойством попарной независимости, вытекает ее свойство кратного перемешивания. Это приводит к следующей задаче: если известна структура самоприсоединений малых порядков, то что можно сказать о самоприсоединениях высоких порядков? Определенный прогресс имеется в случае, когда рассматриваемая система коммутирует только с полиморфизмами достаточно простой структуры (см. [2]), например, с выпуклыми комбинациями автоморфизмов и оператора ортопроекции. В заметке рассматривается новый класс действий, коммутирующих с полиморфизмами более сложной структуры. Для этих действий, названных квазипростыми, установлен упомянутый эффект отсутствия нетривиальных самоприсоединений высоких порядков, что обобщает ряд результатов из [2], [3], [7]. В заметке также установлено, что квазипростое действие и гауссовская система дизъюнктны в смысле Фюрстенберга (заметим,

*Исследования первого автора поддержаны грантом НШ-457.2003.1 
что при этом их спектры могут быть недизъюнктными). Это обобщает результаты из [4] и [9].

1. Пусть $T$ и $T^{\prime}$ - автоморфизмы вероятностных пространств $(X, \mu)$ и $\left(X^{\prime}, \mu\right)$ соответственно. Мера $\nu$ на $X \times X^{\prime}$, проекции которой на сомножители произведения $X \times X^{\prime}$ совпадают с $\mu$ (полиморфизм в терминологии [1]) называется джсоинингом систем $(T, X, \mu)$ и $\left(T^{\prime}, X, \mu\right)$, если $\nu$ инвариантна относительно преобразования $T \times T^{\prime}$. Джойнинг потоков $T_{t}, T_{t}^{\prime}$ определяется аналогично, но здесь требуется инвариантность относительно $T_{t} \times T_{t}^{\prime}$ для всех $t \in \mathbb{R}$.

Говорят, что автоморфизмы $T$ и $T^{\prime}$ дизбюнктны, если они допускают единственный (тривиальный) джойнинг $\mu \times \mu$. Это означает, что существует единственное марковское сплетение унитарных операторов, отвечающих автоморфизмам $T$ и $T^{\prime}$. Этим сплетением является $\Theta$ - ортопроекция на пространство постоянных функций в $L_{2}(X, \mu)$.

Система $(T, X, \mu)$ называется 2 -квазипростой, если для любого эргодического самоприсоединения $\nu \neq \mu \times \mu$ система $(T \times T, X \times X, \nu)$ является изометрическим расширением каждого из своих координатных факторов. Если же для любого эргодического джойнинга $\nu \neq \mu \times \mu$ координатные факторы совпадают, система называется 2 -простой.

«Изометрическое расширение» - синоним расширения с относительно дискретным спектром [10]. Например, система, любое эргодическое самоприсоединение $\nu \neq \mu \times \mu$ которой расположено на графике конечнозначного отображения (в этом случае условные меры $\nu_{x}$ суть конечные суммы точечных мер) будет 2-квазипростой. (Указанным свойством обладают потоки, для которых выполнено свойство Ратнер [6].)

В дальнейшем нам понадобится только следующее свойство изометрических расширений: если система порождена слабо перемешивающим бактором $\mathscr{F}_{1} u$ эргодическими факторами $\mathscr{F}_{2}, \mathscr{F}_{3}$, где $\mathscr{F}_{1}$ и $\mathscr{F}_{2}$ независимы, причем $\mathscr{F}_{2} \vee \mathscr{F}_{3}$ является изометрическим расширением фактора $\mathscr{F}_{2}$, то сумма $\mathscr{F}_{2} \vee \mathscr{F}_{3} u$ фактор $\mathscr{F}_{1}$ независимы (доказательство имеется в [5, Theorem A.3]).

Мы говорим, что автоморфизм $T$ обладает свойством квазипростоты (простоты) порядка $n>2$, если он является 2-квазипростым (2-простым) и любое попарно независимое самоприсоединение $n$ экземпляров автоморфизма $T$ тривиально, т. е. является произведением мер. (Джойнинг $\lambda$ набора автоморфизмов $T_{1}, \ldots, T_{n}, n>2$, называется попарно независимым, если его проекции на любые двумерные грани в декартовом кубе $X_{(1)} \times \cdots \times X_{(n)}$ совпадают с $\left.\mu \times \mu.\right)$

Заметим, что 3-квазипростой автоморфизм $T$ обладает свойством наследственной независимости $(\mathrm{HI})$ : для трех $T$-факторов $\mathscr{F}_{1}, \mathscr{F}_{2}, \mathscr{F}_{3}$, таких, что факторы $\mathscr{F}_{1}$ и $\mathscr{F}_{2}$, а также $\mathscr{F}_{1}$ и $\mathscr{F}_{3}$ независимы, независимы и $\mathscr{F}_{1}$ и $\mathscr{F}_{2} \vee \mathscr{F}_{3}$.

Свойство HI автоморфизма $T$ влечет за собой тривиальность всех попарно независимых самоприсоединений автоморфизма $T$ [7, Theorem 7]. Таким образом, 3-квазипростота эквивалентна квазипростоте всех порядков, что является обобщением аналогичного результата из [3] о 3-простоте.

ТЕОрема 1. Пусть $T_{t}$ является 2-квазипростым слабо перемешиваюшим потоком. Если $\lambda$ - попарно независимое самоприсоединение трех экземпляров потока $T_{t}$, mо $\lambda=\mu \times \mu \times \mu$.

СлЕДСТВИЕ. Для потоков 2 -квазипростота влечет за собой квазипростоту всех порядков. 
Доказательство. Предположим, что $\lambda \neq \mu \times \mu \times \mu$. Декартов куб $X \times$ $X \times X$, на котором определена мера $\lambda$, нам будет удобно записывать в виде $Y_{1} \times Y_{2} \times X_{0}$. Положим $\lambda^{\prime}=\left(\mathrm{Id} \times T_{\varepsilon} \times \mathrm{Id}\right) \lambda$. Рассмотрим новое самоприсоединение $\tilde{\nu}=\lambda \otimes_{Y} \lambda^{\prime}$, которое является относительно независимым произведением мер $\lambda$ и $\lambda^{\prime}$ над фактором $Y=Y_{1} \times Y_{2}$. Напомним, что $\tilde{\nu}$ теперь определена на кубе $Y_{1} \times Y_{2} \times X_{0} \times X_{1}$, а условные меры $\tilde{\nu}_{y}, y \in Y$, по определению суть $\lambda_{y} \times \lambda_{y}^{\prime}$. Полученная мера $\tilde{\nu}$ инвариантна относительно $T_{t} \times T_{t} \times T_{t} \times T_{t}$.

Если для $\varepsilon=0$ проекция относительного произведения равна $\mu \times \mu$, то $\lambda=\mu \times$ $\mu \times \mu$. (На языке марковских операторов это объясняется так: равенство $P^{*} P=$ $\Theta$, где оператор $P$ задается формулой $P f(y)=\int_{X} f(x) d \lambda_{y}(x)$, влечет за собой $P=\Theta$.) Таким образом, для малого $\varepsilon>0$ проекция $\tilde{\nu}$ на $X_{0} \times X_{1}$ не равна мере $\mu \times \mu$. Фиксируем такое число $\varepsilon>0$ и соответствующую меру $\tilde{\nu}$. Из разложения меры $\tilde{\nu}$ на эргодические компоненты получим эргодический джойнинг $\nu_{1}$ на $Y_{1} \times Y_{2} \times X_{0} \times X_{1}$, такой, что $\nu_{1}\left|Y_{1} \times Y_{2} \times X_{0}=\lambda, \nu_{1}\right| Y_{1} \times Y_{2} \times X_{1}=\lambda^{\prime}$, a $\nu_{1} \mid X_{0} \times X_{1}$ является изометрическим расширением меры $\nu_{1} \mid X_{0}$.

По индукции определим на произведении $Y_{1} \times Y_{2} \times X_{0} \times X_{1} \times \cdots \times X_{k}$ последовательность мер $\nu_{k}, k \geqslant 2$, для которых выполнены условия

(1.k) $\nu_{k} \mid Y_{1} \times Y_{2} \times X_{0} \times \cdots \times X_{k-1}=\nu_{k-1}$;

(2.k) $\nu_{k} \mid Y_{1} \times Y_{2} \times X_{1} \times \cdots \times X_{k}=\left(\mathrm{Id} \times T_{\varepsilon} \times S\right) \nu_{k-1}$, где $S$ обозначает правый сдвиг на $X_{0} \times X_{1} \times X_{2} \times \ldots$;

(3.k) $\nu_{k}$ есть изометрическое расширение меры $\nu_{k-1}$.

Положим $\nu_{k+1}$ равной произведению $\nu_{k} \otimes_{Z_{k}}\left(\mathrm{Id} \times T_{\varepsilon} \times S\right) \nu_{k}$ относительно фактора $Z_{k}=Y_{1} \times Y_{2} \times X_{1} \times \cdots \times X_{k}$. Корректность определения меры $\nu_{k+1}$, т. е. совпадение проекций мер $\nu_{k}$ и $\left(\mathrm{Id} \times T_{\varepsilon} \times S\right) \nu_{k}$ на $Z_{k}$, проверяется непосредственно с использованием условий $(1 . \mathrm{k}),(2 . \mathrm{k})$.

Проекция меры $\nu_{k+1}$ на $X_{k} \times X_{k+1}$ совпадает с проекцией на $X_{0} \times X_{1}$ (при естественном отождествлении пространств $X_{k} \times X_{k+1}$ и $\left.X_{0} \times X_{1}\right)$. Так как $X_{k} \times$ $X_{k+1}$ является изометрическим расширением фактора $X_{k}$, то $\left(\cdots \times X_{k}\right) \times X_{k+1}$ является изометрическим расширением фактора $\left(\cdots \times X_{k}\right)$. Свойства $(1 . \mathrm{k}+1)$, $(2 . \mathrm{k}+1)$ джойнинга $\nu_{k+1}$ вытекают из его определения.

Таким образом, на произведении $Y_{1} \times Y_{2} \times\left(X_{0} \times X_{1} \times \ldots\right)$ определена мера $\nu$ как предел мер $\nu_{k}$. По построению мера $\nu$ инвариантна относительно $\operatorname{Id} \times T_{\varepsilon} \times S$, где $S$ - правый сдвиг на произведении $\bar{X}=\cdots \times X_{-1} \times X_{0} \times X_{1} \times \cdots$. Продолжим меру $\nu$ на все пространство $Y_{1} \times Y_{2} \times \bar{X}$ так, чтобы она была инвариантна относительно отображения $\operatorname{Id} \times T_{\varepsilon} \times S$. При этом проекция меры $\nu$ на $Y_{1} \times \bar{X}$ совпадает с произведением проекций на $Y_{1}$ и $\bar{X}$ (здесь мы воспользовались тем, что изометрические расширения сохраняют свойство независимости факторов). Аналогичным свойством обладает проекция меры $\nu$ на $Y_{2} \times \bar{X}$. Поскольку автоморфизм $T_{\varepsilon}$ слабо перемешивающий, из равенства $\nu=\left(\operatorname{Id} \times T_{\varepsilon} \times S\right) \nu$ вытекает, что $\nu$ является произведением мер (см. [2, лемма 2.2]). Так как $\nu \mid Y_{1} \times Y_{2} \times X_{0}=\lambda$, получим $\lambda=\mu \times \mu \times \mu$.

Фактически мы доказали следующее утверждение.

ТЕОремА 2. Пусть $\lambda$ - попарно независимый джсоининг слабо перемешивающих автоморфизмов $G, G^{\prime}$ и 2-квазипростого автоморбизма $T$, причем $G^{\prime}$ вкладьвается в поток. Тогда $\lambda=\mu \times \mu \times \mu$.

(Отличие в доказательстве состоит в замене $T_{\varepsilon}$ на $G_{\varepsilon}^{\prime}$.)

2. Второй из авторов в работе [8] показал, что гауссовский автоморфизм и простой автоморфизм, коммутирующий только со своими степенями, дизъ- 
юнктны. В [4] доказано, что простой (всех порядков) автоморфизм и гауссовский автоморфизм дизъюнктны. В [9] этот результат обобщен: простой автоморфизм дизъюнктен с бесконечно делимым автоморфизмом, вложенным в поток (гауссовские автоморфизмы обладают этим свойством).

Будем для технического удобства записывать $(G, X)$ как прямое произведение $\prod_{i=1}^{n}\left(G_{i}, X_{i}\right)$ «маленьких» факторов $\left(G_{i}, X_{i}\right)$. (На самом деле вместо произведения рассматривается сумма независимых факторов.)

Система $(G, X, \mu)$ называется бесконечно делимой, если для любой окрестности $U$ оператора $\Theta$ (ортопроекции на пространство констант в $\left.L_{2}(X, \mu)\right)$ найдется набор независимых факторов, порождающих систему $(G, X, \mu)$, такой, что все проекторы на пространства, отвечающие этим факторам, лежат в $U$ (здесь рассматривается слабая операторная топология).

Гауссовские автоморфизмы обладают следующим более сильным свойством. Систему $(G, X, \mu)$ назовем равномерно делимой, если для любой окрестности $V$ тождественного оператора в $L_{2}(X, \mu)$ можно найти такую факторизацию $\prod_{i=1}^{n}\left(G_{i}, X_{i}\right)$, что для всех $k=1, \ldots, n$ ортопроекции на пространства $L_{2}\left(\prod_{i, i \neq k} X_{i}\right)$ лежат в $V$. (Выбрасывая из произведения один сомножитель, мы «мало» теряем.)

ТеоремА 3. Пусть автоморфизм $T$ является 2-квазипростым, а автоморбизм $G$ - слабо перемешиваюшим. Если $G$ бесконечно делим и вкладьвается в поток, то $G$ и $T$ дизгюнктнъ.

ЛЕмма 4. Пусть $\nu$ - эргодический джсойнинг 2-квазипростой системы $(T, X)$ и слабо перемешивающей системъ $\left(G_{1} \times G_{2}, Y_{1} \times Y_{2}\right)$. Если $X$ и $Y_{1}$ не являются независимыми относительно меры $\nu$, то $Y_{2}$ u $X \times Y_{1}$ независимы.

ЗАмЕЧАНИЕ. Иными словами лемма утверждает, что для марковского опеpaтора $J: L_{2}(X) \rightarrow L_{2}\left(Y_{1} \times Y_{2}\right)$, отвечающего джойнингу $\nu$, условие $\operatorname{Im} J \cap$ $L_{2}\left(Y_{1}\right) \neq\{$ Const $\}$ влечет за собой $\operatorname{Im} J \subset L_{2}\left(Y_{1}\right)$. Последнее переписывается следующим образом: для ортопроекции $P$ на пространство $L_{2}\left(Y_{1}\right)$ условие $P J \neq \Theta$ влечет за собой $P J=J$.

ДокаЗательство леммы 4. Рассмотрим на $X \times Y_{1} \times Y_{2} \times X^{\prime}\left(X=X^{\prime}\right)$ эргодическую компоненту относительного произведения $\nu \otimes_{Y_{1} \times Y_{2}} \nu^{\prime}$, где $\nu^{\prime}=$ $\left.\nu\right|_{X^{\prime} \times Y_{1}} \times\left.\nu\right|_{Y_{2}}$ (этой мере отвечает оператор $\left.P J\right)$.

Если $X$ и $X^{\prime}$ зависимы, то $X \times X^{\prime} \times Y_{1}$ и $Y_{2}$ независимы, поскольку $X^{\prime} \times Y_{1} \perp$ $Y_{2}$, а $X \times X^{\prime} \times Y_{1}$ является изометрическим расширением пространства $X^{\prime} \times Y_{1}$. Таким образом, $X \times Y_{1} \perp Y_{2}$.

Пусть проекция $\nu \otimes_{Y_{1} \times Y_{2}} \nu^{\prime}$ на $X \times X^{\prime}$ равна $\mu \otimes \mu$, т. е. $J^{*} P J=\Theta$. Так как $J^{*} P J=J^{*} P^{*} P J$, получим $J^{*} P^{*} P J=\Theta$, что равносильно равенству $P J=\Theta$. Последнее означает, что $X \perp Y_{1} \times Y_{2}$ относительно $\nu^{\prime}$, а следовательно, $X \perp Y_{1}$ относительно $\nu$.

ДокАЗАТЕЛЬСтво теоремы 3. Пусть марковский оператор $J$ сплетает $T$ и $G$, а $G$ факторизован в произведение независимых факторов $\prod_{i=1}^{n}\left(G_{i}, X_{i}\right)$. Тогда в силу леммы 4

(а) или в ортогональном дополнении к постоянным функциям образ оператора $J$ ортогонален всем пространствам $L_{2}\left(X_{i}\right)$ (здесь $L_{2}\left(X_{i}\right)$ обозначает подпространство, отвечающее $i$-му фактору),

(b) или образ оператора $J$ лежит в одном из пространств $L_{2}\left(X_{i}\right)$. 
Случай (а) означает, что джойнинг, отвечающий оператору $J$, является попарно независимым, а следовательно, в силу теоремы 2 тривиальным.

ЗАмЕчАниЕ. Если автоморфизм $T$ является 3-квазипростым, то попарно независимый джойнинг автоморфизма $T$ с любыми автоморфизмами $G_{1}, G_{2}$ является тривиальным.

Если же случай (а) не возникает, то получаем, что $J=P J$, где $P$ обозначает ортопроекцию на пространство $L_{2}\left(X_{i}\right)$ из п. (b). Производя все более мелкое деление автоморфизма $G$, получим последовательность ортопроекций $P_{N} \rightarrow \Theta$, таких, что $J=P_{N} J$. Следовательно, $J=\Theta$.

ТеоремА 5. Пусть автоморфизм $T$ является 2-квазипростым, а автоморбизм $G$ - слабо перемешиваюшим и равномерно делимым. Тогда $G$ и $T$ дизгюнктны.

ДокАзАтельство. Пусть марковский оператор $J \neq \Theta$ сплетает унитарные операторы, индуцированные автоморфизмами $T$ и $G$. В силу равномерной делимости найдем такую факторизацию $(G, X)=\prod_{i=1}^{n}\left(G_{i}, X_{i}\right)$ в произведение $n$ факторов, для которой $\breve{P}_{k}$, ортопроекции на пространства $L_{2}\left(\prod_{i \neq k} X_{i}\right)$, настолько близки к тождественному оператору $I$, что удовлетворяют условиям $\breve{P}_{k} J \neq \Theta, k=1, \ldots, n$. Положим $Y_{1}=\prod_{i \neq k} X_{i}, Y_{2}=X_{k}$ и применим лемму 4. Имеем $J=\breve{P}_{k} J$ для всех $k=1, \ldots, n$. Получим $J=\breve{P}_{1} \breve{P}_{2} \cdots \breve{P}_{n} J=\Theta J=\Theta$.

Результаты заметки в основном получены авторами во время пребывания первого из них в Лаборатории вероятностей VI Парижского университета в 2002 г., ее написание завершено во время пребывания второго из авторов в Московском университете в январе 2005 г.

Авторы признательны М. Леманчику и А. дель Юнко, любезно приславшим препринт, содержащий элементы общей теории квазипростых систем.

ЗАмечАниЕ. А. И. Даниленко построил квазипростые автоморфизмы качественно нового типа по сравнению с ранее известными. Его действия и любое простое действие дизъюнктны.

\section{ЛитерАтУРА}

[1] А. М. Вершик, Зап. науч. сем. ЛОМИ, 72 (1977), 26-62. [2] В. В. Рыжиков, Maтем. сб., 188:2 (1997), 67-94. [3] E. Glasner, B. Host, D. Rudolph, Israel J. Math., 78 (1992), 131-142. [4] A. del Junco, M. Lemanczyk, Studia Math., 133 (1999), 249-256. [5] J. L. King, J.-P. Thouvenot, J. Analyse. Math., 56 (1991), 211-230. [6] M. Ratner, Ann. of Math. (2), 115:3 (1982), 597-614. [7] V. V. Ryzhikov, J. Dynam. Control Systems, 3:1 (1997), 111-127. [8] J.-P. Thouvenot, in: Ergodic Theory and Its Connections with Harmonic Analysis (Proceedings of the Alexandria 1993 Conference), London Math. Soc. Lecture Note Ser., vol. 205, Cambridge Univ. Press, Cambridge, 1995, 207-235. [9] J.-P. Thouvenot, Colloq. Math., 84/85, part 2 (2000), 481-483. [10] R. Zimmer, Ill. J. Math., 20 (1976), 555-588.

Московский государственный университет им М. В. Ломоносова e-mail: vryz@mech.math.msu.su 17 февраля 2005 г.

6-й Парижский университет П. и М. Кюри e-mail: kalikow@ccr.jussieu.fr 